\title{
LOCAL MODULI \\ FOR MEROMORPHIC DIFFERENTIAL EQUATIONS
}

\author{
BY DONALD G. BABBIT'T AND V. S. VARADARAJAN
}

1. Introduction. This note announces results concerning the parametrization, in the sense of (local) moduli, of the equivalence classes of systems of meromorphic differential equations of the form

$$
d u / d z=A u
$$

near an irregular singular point (assumed to be $z=0$ ). Here $u$ is an $n$ component column vector, $A$ is an $n \times n$ matrix of meromorphic functions, and equivalence of systems defined by matrices $A$ and $B$ means that there is a meromorphic invertible $n \times n$ matrix $x$ such that

$$
x[A] \stackrel{\text { def }}{=} x A x^{-1}+(d x / d z) x^{-1}=B
$$

near $z=0$. If $\mathcal{F}_{\text {cgt }}$ (resp. $\mathcal{F}$ ) is the field of quotients of the ring of convergent (resp. formal) power series in $z$ with coefficients in $\mathbf{C},(* *)$ defines an action of $\mathrm{GL}\left(n, \mathcal{F}_{\text {cgt }}\right)$ on $\mathfrak{g l}\left(n, \mathcal{F}_{\text {cgt }}\right)$, reflecting the fact that $(*)$ goes over to the system $d v / d z=B v$ under the substitution $v=x u$; replacing $\mathcal{F}_{\text {cgt }}$ by $₹$ leads to the notion of formal equivalence. We note that for any commutative ring $R$ (with unit) equipped with a derivation $D,(* *)$ defines an action of $\operatorname{GL}(n, R)$ on $\mathfrak{g l}(n, R)$, with $D$ replacing $d / d z$; if $R$ is a suitably restricted ring of Laurent series in $z$ with coefficients in the ring of convergent power series in $d$ variables and $D=d / d z$, we obtain the notion of equivalence of analytic families of systems $(*)$ depending on $d$ parameters, which is basic to the theory of local moduli (cf. [BV2]).

One parametrizes the equivalence classes of systems $(*)$ in two steps. The first step is the classification up to formal equivalence, i.e., the description of the orbit space $\operatorname{GL}(n, \mathcal{F}) \backslash \mathfrak{g l}(n, \mathcal{F})$; the second step is to fix a formal class $\Omega$ with $\Omega_{\text {cgt }} \stackrel{\text { def }}{=} \Omega \cap \mathfrak{g l}\left(n, \mathcal{F}_{\text {cgt }}\right) \neq \emptyset$, and to classify the systems $(*)$ in $\Omega_{\text {cgt }}$ up to equivalence, i.e., to describe the orbit space $\operatorname{GL}\left(n, \mathcal{F}_{\text {cgt }}\right) \backslash \Omega_{\text {cgt }}$. The description of $\operatorname{GL}(n, \mathcal{F}) \backslash \mathfrak{g l}(n, \mathcal{F})$; goes back to Hukuhara and Turrittin (see [BV1] for extensive references) and is based on the notion of a canonical form. The classical method of studying the second question is based on the technique of Stokes lines and Stokes multipliers $[\mathbf{B i}, \mathbf{J}]$. Recently this has been examined from a more modern, and essentially cohomological, point of view, notably by Malgrange [Ma1, Ma2], Sibuya [S], and Deligne (cf. [Be]). The present note continues this theme by studying the equivalence of analytic families of systems $(*)$ and is based in a fundamental way on the theory of formal equivalence over general rings developed in [BV2].

Received by the editors April 4, 1984 and, in revised form, August 15, 1984 1980 Mathematics Subject Classification. Primary 34A20. 
For our purposes we define a canonical form to be an element of $\mathfrak{g l}\left(n, \mathcal{F}_{\text {cgt }}\right)$ of the type

$$
B=D_{r_{1}} z^{r_{1}}+\cdots+D_{r_{m}} z^{r_{m}}+z^{-1} C,
$$

where (a) $r_{1}<r_{2}<\cdots<r_{m}<-1$, the $r_{i}$ being integers, (b) $C, D_{r_{1}}, \ldots, D_{r_{m}}$ are elements of $\mathfrak{g l}(n, \mathbf{C})$ that commute with each other, (c) the $D_{r_{2}}$ are nonzero and semisimple, and (d) the real parts of all the eigenvalues of $C$ are in $[0,1)$ (if $m=0, B=z^{-1} C$ ). For $\Omega$ we take the $\operatorname{GL}(n, \mathcal{F})$-orbit of $B$. We put $\Omega(B)=\Omega_{\text {cgt }}$ and write $X(B)$ for the space $\operatorname{GL}\left(n, \mathcal{F}_{\text {cgt }}\right) \backslash \Omega(B)$. Our main results show (cf. $\S 3$ ) that $X(B)$ may be viewed in a natural way as a space of the form $G_{B} \backslash H(B)$, where $H(B)$ is an algebraic variety isomorphic to an affine space $\mathbf{C}^{d}$ and $G_{B}$ is an algebraic subgroup of $\operatorname{GL}(n, \mathbf{C})$ acting morphically on $H(B)$, and that "local moduli" exist at the "good" points of this quotient space: the restriction to "good" points is essential even in the simplest cases. Our results may thus be viewed as a description of the analytic deformations of the meromorphic differential equations $d u / d z=A u$ when one fixes all the formal invariants of the equation, at least when the point of $H(B)$ defined by $A$ is "smooth and stable".

2. The Stokes sheaf $\mathrm{St}_{B}$ and the identification $\mathrm{GL}\left(n, \mathcal{F}_{\text {cgt }}\right) \backslash \Omega(B) \approx$ $G_{B} \backslash H^{1}\left(\operatorname{St}_{B}\right)$. Fix $B$ as in $\S 1$ and let $\Psi=\exp \left\{\sum_{1 \leq j \leq m}\left(r_{j}+1\right)^{-1} D_{r_{j}} z^{r_{j}+1}\right\}$. The Stokes sheaf $\mathrm{St}_{B}$ is the sheaf of (in general noncommutative) groups defined on the unit circle $T$ as follows: for any open subset $U$ of $T, \operatorname{St}_{B}(U)$ is the group of holomorphic maps of the sector $\Gamma(U)=\left\{\left.z \in \mathbf{C}^{\times}|z| z\right|^{-1} \in U\right\}$ into $\mathrm{GL}(n, \mathbf{C})$ such that

$$
\begin{gathered}
\Psi g \Psi^{-1} \sim 1(\Gamma(U)), \\
d g / d z=z^{-1}[C, g] \quad \text { on } \Gamma(U) .
\end{gathered}
$$

Here, the notation $\sim 1(\Gamma(U))$ in (a) means that, for any closed arc $U^{\prime} \subset U$ and any $r \geq 1$, we have $\Psi g \Psi^{-1}-1=O\left(|z|^{r}\right)$ as $z \rightarrow 0$ in $\Gamma\left(U^{\prime}\right)$, the $O$ being uniform in $\Gamma\left(U^{\prime}\right)$. If $U$ is an $\operatorname{arc} \neq T$ and $z_{U}^{C}=\exp \left(\log _{U} z \cdot C\right)$, where $\log _{U}$ is a branch of the logarithm on $\Gamma(U)$, the map $g \rightarrow z_{U}^{-C} g z_{U}^{C}$ takes $\operatorname{St}_{B}(U)$ onto a unipotent algebraic subgroup of $\operatorname{GL}(n, \mathbf{C})$ which is independent of the choice of the logarithm. So all the $\mathrm{St}_{B}(U)$ become unipotent algebraic groups in a natural way. Consequently, if $\mathfrak{U}=\left(U_{i}\right)$ is a finite open covering of $T$ by $\operatorname{arcs} \neq T$, the set $C\left(\mathfrak{U}: \mathrm{St}_{B}\right)=\prod_{i} \mathrm{St}_{B}\left(U_{i}\right)$ becomes a unipotent algebraic group, the set $Z^{1}\left(\mathfrak{U}: \mathrm{St}_{B}\right)$ of Cech 1-cocycles becomes an affine variety on which $C\left(\mathfrak{U}: \mathrm{St}_{B}\right)$ acts, and the space of orbits can be naturally identified with $H^{1}\left(\mathfrak{U}: \mathrm{St}_{B}\right)$. As usual, $H^{1}\left(\mathrm{St}_{B}\right)$ is the union of all the $H^{1}\left(\mathfrak{U}: \mathrm{St}_{B}\right)$ as $\mathfrak{U}$ varies over the coverings as above. If $G_{B}$ is the centralizer of $C, D_{r_{1}}, D_{r_{2}}, \ldots, D_{r_{m}}$ in $\mathrm{GL}(n, \mathbf{C}), G_{B}$ acts on each $\operatorname{St}_{B}(U)$ by $g, u \rightarrow g[u]=g u g^{-1}$, and hence on $H^{1}\left(\mathrm{St}_{B}\right)$. Our starting point is the following variant of a theorem of SibuyaMalgrange ([S, Ma1]; cf. also [Maj]).

Proposition 1. There is a natural map $\theta$ from $\Omega(B)$ to $G_{B} \backslash H^{1}\left(\mathrm{St}_{B}\right)$ that is constant on the orbits of $\mathrm{GL}\left(n, \mathcal{F}_{\mathrm{cgt}}\right)$ in $\Omega(B)$ and induces a bijection of $X(B)$ with $G_{B} \backslash H^{1}\left(\mathrm{St}_{B}\right)$.

3. The main theorems. By an analytic family $a$ in $\mathcal{F}_{\text {cgt }}$ we mean a family $\{a(\lambda)\}\left(\lambda \in \Delta^{q}\right)$, where $\Delta^{q}$ is a polydisc in $\mathbf{C}^{q}$ centered at the origin, 
$a(\lambda) \in \mathcal{F}_{\text {cgt }}$ for all $\lambda \in \Delta^{q}$, and there is an integer $r \geq 1$ such that, for some holomorphic function $a^{\prime}$ on $\Delta^{q} \times\{z|| z \mid<\varepsilon\}, a(\lambda)$ is the element of $F_{\text {cgt }}$ defined by $z^{-r} a^{\prime}(\lambda: z)$. This leads in an obvious way to the notion of analytic families in $\mathfrak{g l}\left(n, \mathcal{F}_{\mathrm{cgt}}\right)$ and in $\mathrm{GL}\left(n, \mathcal{F}_{\mathrm{cgt}}\right)$. If $A$ and $A_{1}$ are analytic families in $\mathfrak{g l}\left(n, \mathcal{F}_{\mathrm{cgt}}\right)$ defined over $\Delta^{q}$, they are called equivalent if there is an analytic family $x$ in $\operatorname{GL}\left(n, \mathcal{F}_{\text {cgt }}\right)$ such that $x(\lambda)[A(\lambda)]=A_{1}(\lambda)$ for all $\lambda$ in some neighbourhood of the origin. An analytic family $A$ in $\mathfrak{g l}\left(n, \mathcal{F}_{\text {cgt }}\right)$ is said to be in $\Omega(B)$ if $A(\lambda)$ is in $\Omega(B)$ for all $\lambda$ in some neighbourhood of the origin.

Let $\Sigma$ be the set of Laurent polynomials $\sigma=\sum_{1 \leq j \leq m} a_{j} z^{r_{j}}$, where $a_{j}$ is any eigenvalue of $D_{r_{j}}, 1 \leq j \leq m$. For $\sigma, \tau \in \Sigma$ with $\sigma \neq \tau$, let $q=$ $q(\sigma, \tau) \leq-2$ be the order of $\sigma-\tau, c_{q}$ the coefficient of $z^{q}$ in $\sigma-\tau$, and let $S(\sigma, \tau)$ be the (finite) set of rays in $\mathbf{C}^{\times}$where $\operatorname{Re}\left(c_{q} z^{q}\right)$ vanishes. The rays belonging to $\bigcup_{\sigma, \tau \in \Sigma, \sigma \neq \tau} S(\sigma, \tau)$ are called the Stokes lines of $B$. Let $\mathfrak{T}(B)$ denote the collection of all finite coverings $\mathfrak{U}=\left(U_{i}\right)$ of $T$ by open $\operatorname{arcs}$ of length $\leq \pi /\left(\left|r_{1}\right|-1\right)$ with the restriction that the ends of the arcs of length equal to $\pi /\left(\left|r_{1}\right|-1\right)$ are not on any Stokes line.

THEOREM 1. (i) $H^{1}\left(\mathrm{St}_{B}\right)$ can be given the structure of an algebraic variety which is natural in the following sense: for any $\mathfrak{U} \in \mathfrak{T}(B), C\left(\mathfrak{U}: \mathrm{St}_{B}\right)$ acts freely on $Z^{1}\left(\mathfrak{U}: \mathrm{St}_{B}\right), H^{1}\left(\mathfrak{U}: \mathrm{St}_{B}\right)=H^{1}\left(\mathrm{St}_{B}\right)$, and $H^{1}\left(\mathrm{St}_{B}\right)$ is the geometric quotient of $Z^{1}\left(\mathfrak{U}: \mathrm{St}_{B}\right)$ for this action (see [MF] for the notion of geometric quotient); moreover, there is a global cross section for this action.

(ii) $H^{1}\left(\mathrm{St}_{B}\right)$ is isomorphic to the affine space $\mathbf{C}^{d}$, where $d$ is the irregularity of $B$ in the sense of Malgrange (cf. [Be, pp. 233, 238]).

(iii) The action of $G_{B}$ on $H^{1}\left(\mathrm{St}_{B}\right)$ is algebraic.

A point $\gamma \in H^{1}\left(\mathrm{St}_{B}\right)$ is called $G_{B}$-smooth if there exists a $G_{B}$-invariant open set $U$ containing $\gamma$ such that the geometric quotient $G_{B} \backslash U$ exists in the category of complex analytic manifolds. Let $H^{1}\left(\mathrm{St}_{B}\right)^{\mathrm{sm}}$ be the $G_{B}$-invariant open set of $G_{B}$-smooth points. Let $Y=G_{B} \backslash H^{1}\left(\mathrm{St}_{B}\right), \pi$ the natural map $H^{1}\left(\mathrm{St}_{B}\right) \rightarrow Y$, and $Y^{\mathrm{sm}}=\pi\left(H^{1}\left(\mathrm{St}_{B}\right)^{\mathrm{sm}}\right) ; Y$ is given the quotient topology. The sheaf of $G_{B}$-invariant analytic functions on $H^{1}\left(\mathrm{St}_{B}\right)$ defines a sheaf on $Y$ and converts $Y$ into a ringed space; and $Y^{\mathrm{sm}}$ is the open subset of points around which this ringed space looks like a complex manifold of dimension $r=d-\delta$, where $\delta$ is the maximum dimension of the $G_{B}$-orbits in $H^{1}\left(\mathrm{St}_{B}\right)$.

THEOREM 2. Fix $\gamma \in H^{1}\left(\mathrm{St}_{B}\right)^{\mathrm{sm}}$. Let $A$ be an analytic family of elements in $\Omega(B)$ defined over $\Delta^{q}$ such that $\theta(A(0))=\pi(\gamma)$. Then $\mu(A): \lambda \rightarrow \theta(A(\lambda))$ is an analytic map of a neighbourhood of the origin into a neighbourhood of $\pi(\gamma)$. If $A_{1}$ is another analytic family in $\Omega(B)$ defined over $\Delta^{q}$ such that $\mu(A)=\mu\left(A_{1}\right)$ in a neighbourhood of the origin, then $A$ and $A_{1}$ are equivalent.

The proof of this theorem relies heavily on one of the main results of [BV2].

THEOREM 3. Let $r$ be as defined earlier. Then we can find an analytic family in $\Omega(B)$ defined over $\Delta^{r}$ such that $\mu(A)$ is an analytic isomorphism of a neighbourhood of the origin in $\Delta^{r}$ with a neighbourhood of the point $\pi(\gamma)$. Any such family is universal in the following sense. If $A_{1}$ is any analytic family in $\Omega(B)$ defined over $\Delta^{q}$ with $\theta\left(A_{1}(0)\right)=\pi(\gamma)$, we can find an analytic map 
$\alpha: \Delta^{\prime q} \rightarrow \Delta^{\prime r}$ (primes denote concentric polydiscs) vanishing at the origin such that the families $A_{1}$ and $A \circ \alpha$ are equivalent.

If $C$ is semisimple, $G_{B}$ is reductive, so we are in the paradigm of Mumford [MF]. Let us call a point $\gamma \in H^{\mathbf{1}}\left(\mathrm{St}_{B}\right)$ stable if its $G_{B}$-orbit is closed and has dimension $\delta$, and let $H^{1}\left(\mathrm{St}_{B}\right)^{\mathrm{s}}$ be the set of stable points; it is $G_{B}$-invariant and Zariski open. The statement that $H^{1}\left(\mathrm{St}_{B}\right)^{\mathrm{s}} \neq \emptyset$ is equivalent to saying that the action of $G_{B}$ on $H^{1}\left(\mathrm{St}_{B}\right)$ is generically stable (cf. [MF, p. 154]).

THEOREM 4. Suppose $C$ is semisimple and $H^{1}\left(\mathrm{St}_{B}\right)^{\mathbf{s}} \neq \emptyset$. Then $Y^{\mathbf{s}}=$ $G_{B} \backslash H^{1}\left(\mathrm{St}_{B}\right)^{\mathrm{s}}$ is an irreducible quasi-affine variety of dimension $r$. If $\Gamma$ is the set of points $\gamma$ in $H^{1}\left(\mathrm{St}_{B}\right)^{\mathrm{s}}$ such that $\pi(\gamma)$ is a simple point in $Y^{\mathrm{s}}$, then $\Gamma \subset H^{1}\left(\mathrm{St}_{B}\right)^{\mathrm{sm}}, \Gamma$ is dense in $H^{1}\left(\mathrm{St}_{B}\right)$, and $G_{B} \backslash \Gamma$ is a complex manifold of dimension $r$.

Already in simple examples such as the Bessel and Whittaker equations, nonsmooth and smooth nonstable points exist. In general, $G_{B} \backslash H^{1}\left(\mathrm{St}_{B}\right)^{\mathrm{sm}}$ will not be separated. When $B$ is such that the restriction of $C$ to each spectral subspace of $\left(D_{r_{1}}, \ldots, D_{r_{m}}\right)$ has a simple spectrum, then stable points exist, $P G_{B}=G_{B} / \mathbf{C}^{\times}$acts generically freely on $H^{1}\left(\mathrm{St}_{B}\right)$, and $r=d-n+1$.

\section{REFERENCES}

[Be] D. Bertrand, Travaux récents sur les points singuliers des équations différentielles linéaires, Sem. Bourbaki 1978/79, Exp. 525-542, Lecture Notes in Math., no. 770, Springer-Verlag, 1980.

[Bi] G. D. Birkhoff, Collected mathematical papers, Vol. 1, Dover, New York, 1968.

[BV1] D. G. Babbitt and V. S. Varadarajan, Formal reduction theory of meromorphic differential equations: a group theoretic view, Pacific J. Math. 108 (1983), 1-80.

[BV2] _ Deformations of nilpotent matrices over rings and reduction of analytic families of meromorphic differential equations, Mem. Amer. Math. Soc. (to appear).

[J] W. Jurkat, Meromorphe Differentialgleichungen, Lecture Notes in Math., no. 637, Springer-Verlag, 1978.

[Ma1] B. Malgrange, Remarques sur les équations différentielles à points singuliers irréguliers, Lecture Notes in Math., no. 712, Springer-Verlag, 1979.

[Ma2] __ La classification des connexions irréguliers à une variable, Mathématique et Physique: Sém. École Norm. Sup. 1979-1982, Birhäuser, 1983.

[Maj] H. Majima, Analogues of Cartan's decomposition theorem in asymptotic analysis, Funkcial. Ekvac. 26 (1983), 131-154.

[MF] D. Mumford and J. Fogarty, Geometric invariant theory, 2nd Enl. Ed., SpringerVerlag, 1982.

[S] Y. Sibuya, Stokes phenomena, Bull. Amer. Math. Soc. 83 (1977), 1075-1077.

\footnotetext{
DEPARTMENT OF MATHEMATICS, UNIVERSity OF CALIFORNIA, LOS ANGElES, CALIFORNIA 90024
} 